\title{
Evaluación de Competencias Transversales en un entorno 3.0: Lessons (Sakai)
}

Héctor Moreno-Ramón, Sara Ibáñez-Asensio, Ignacio Guillén Guillamón, Miguel Ferrando Bataller, Salva Calvet Sanz, Marta Cabedo Fabrés, Ana I. Jiménez Belenguer y Alfonso Martínez García.

${ }^{a}$ GRIPAU, Grupo Interdisciplinar para el Aprendizaje Universitario. Universitat Politècnica de València (hecmora@prv.upv.es )

\begin{abstract}
Transversal skills have led to restructuring subjects, methodology and materials in Higher Education. The Universitat Politècnica of Valencia $(U P V)$ has begun to apply and assess these skills in their degrees where the students are the center of the learning process. Faced with these problems, the following study aims to facilitate and test the assessment of tranversal skills in the UPV through an advanced tool (Lessons) that has been developed by Sakai, and it is available to the entire university community within the PoliformaT platform. This tool allows not only the evaluation of skills in a traditional way (student-teacher), but also establishes an environment based on the philosophy of web 3.0 where 3 types of learning interactions are produced (student-teacher, teacher-student and Studentstudent). In addition, it allows to redirect the students according to theis knowledge and allows self-evaluation before the activity development. The experience was carried out on 730 students who belong to 11 subjetcs in different degrees/master/doctorate courses. Outcomes registered in Lessons expressed that more than $80 \%$ of them believe that they should be selfassessed at the beginning. In this sense, more than $70 \%$ of the students express that they have achieved the transversal skill, although this outcomes contrast with the teachers' evaluation, which reduce the level of skill achievement.
\end{abstract}

Keywords: Transversal skill, Lessons, PoliformaT, ICTs. 


\begin{abstract}
Resumen
Las competencias transversales han provocado una reestructuración de materias, metodologías y materiales en la Educación Superior. La Universitat Politècnica de Valencia (UPV) ha comenzado a aplicar y evaluar estas competencias en sus grados, donde los alumnos son el centro del proceso de aprendizaje. Frente a esta situación, el presente estudio tiene como objetivo facilitar y probar la evaluación de competencias transversales en la UPV a través de una herramienta avanzada (Lessons) que ha sido desarrollada por Sakai, y está disponible para toda la comunidad universitaria dentro de la plataforma PoliformaT. Esta herramienta permite no sólo la evaluación de competencias de manera tradicional (estudianteprofesor), sino que también establece un entorno basado en la filosofia de la web 3.0, donde se producen 3 tipos de interacciones de aprendizaje (estudiante-profesor, profesor- estudiante y estudiante-estudiante). Además, permite reorientar a los estudiantes según su conocimiento además de la autoevaluación antes del desarrollo de la actividad. La experiencia se llevó a cabo en 730 estudiantes que pertenecen a 11 asignaturas en diferentes grados / master y doctorado. Los resultados registrados en Lessons reflejaron que más del $80 \%$ de los alumnos vieron idóneamente el uso de Lessons para la autoevaluación inicial. En este sentido, más del 70\% de los estudiantes creían tener adquirida la competencia transversal, aunque estos resultados contrastaron con la evaluación de los profesores, que indicaron que no todos los alumnos tenían un nivel de adquisición de la competencia tan elevado como ellos creían.
\end{abstract}

Palabras clave: Competencias transversales, Lessons, PoliformaT, TIC.

\title{
Introducción
}

En los últimos años, el sistema de enseñanza universitario ha derivado hacia un modelo basado y centrado en el alumno, siendo éste la figura en torno a la cual gira la planificación de las asignaturas. El estudiante pasa de ser un "objeto inmóvil" a ser el motor propio del mismo proceso educativo. Así pues, el alumno aprende cuando construye nuevos conocimientos o perfecciona los que ha adquirido, reordenándolos, reflexionando, razonando y obteniendo sus propias conclusiones (Bain, 2007). De este modo se reemplaza la metodología tradicional por un sistema combinado de enseñanza-aprendizaje en el que el alumno se siente motivado y partícipe de sus avances (Lucena, 2015).

Este cambio de roles hizo replantearse de arriba abajo la Educación Superior y de acuerdo con Fernández (2006) supuso un gran cambio cultural para la Universidad como institución educativa. Preguntas como: ¿Cómo estamos enseñando?, ¿Qué pide la sociedad de nuestro

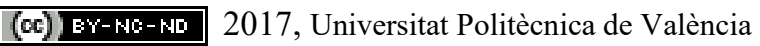


sistema educativo? ¿Satisfacemos sus necesidades? ¿Qué podemos ofrecer de forma única y diferenciada?, etc., fueron la clave para una vorágine de cambios que alumnado y profesorado comienzan a asimilar claramente en la actualidad.

En este nuevo contexto, se descubrió que el mundo laboral no requería tanto "conocimiento", sino más bien precisaba de una serie de habilidades y destrezas que carecían los egresados y que les impedía el buen desempeño de las labores en su puesto de trabajo. De ahí nace la educación basada en competencias, cuya finalidad es dar respuesta a las exigencias de la sociedad y ofrecer una educación de calidad no solamente basada en la enseñanza (Córdova et al., 2015; Munuera y Navarro, 2015).

La Universitat Politècnica de València no se ha mantenido ajena a este proceso y ha hecho suya la filosofía docente derivada de estos nuevos paradigmas. Dentro del plan estratégico UPV2020, la Universidad ha lanzado un proyecto de competencias transversales que "pretende acreditar las competencias transversales UPV a los estudiantes egresados en cualquiera de los títulos oficiales impartidos en la Universitat Politècnica de València" (UPV, 2014). Conscientes que las competencias específicas se trabajan, son adquiridas por los estudiantes y certificadas mediante la superación de las diferentes materias de los planes de estudio, no ocurría lo mismo con las competencias transversales incluidas en los planes de estudio. Éstas no se trabajaban sistemáticamente y no se evaluaban. Esto implicaba que no se garantizaba su adquisición al lanzar al mundo laboral a los egresados en la UPV. De este modo, si la Universidad planteaba unos cánones de calidad y en cambio no se podía acreditar dichas competencias, existía un claro desajuste asociado al proceso de enseñanzaaprendizaje. Con el plan UPV2020, junto al trabajo colaborativo entre el Instituto de Ciencias de la Educación (ICE) y el profesorado de la Universidad, dicha falta de control se está subsanando. La creación de grupos de trabajo que han desarrollado rúbricas para la evaluación de competencias o la reflexión profunda sobre la aplicación de éstas en el quehacer diario de alumnos y profesores, ha dado lugar a una mejora sustancial de dicho proceso de enseñanza-aprendizaje.

El Grupo Interdisciplinar para el Aprendizaje Universitario (GRIPAU) es un equipo multidisciplinar formado por profesores que imparten docencia en diferentes escuelas de la UPV y que desde hace años trabaja para desarrollar y evaluar competencias transversales con el objetivo de promover la excelencia del alumnado. Para ello GRIPAU, participa activamente de los grupos de trabajo de las 13 competencias transversales definidas por la UPV en su proyecto institucional.

La tecnología ha modificado los hábitos sociales y culturales, propiciando la aparición de nuevos métodos de enseñanza-aprendizaje que se basan en las tecnologías de la información y comunicación (TIC's). Es una obviedad como subraya Lucena (2015) que las acciones que se desarrollen desde el punto de vista educativo no pueden dejar de lado el nuevo contexto digital y su papel dentro del proceso formativo. Una facilidad que ofrecen las TIC's de cara al docente es el papel de tutelaje que el profesor puede ejercer sobre el proceso de aprendizaje, puesto que el alumno, dispone de herramientas para su propia 
preparación. Con ellas, el profesor es un gestor de contenidos, siendo las TIC's un elemento dinamizador para el desarrollo de las diferentes competencias (Mezarina et al., 2014). La motivación intrínseca del alumno (conocedor de este tipo de herramientas) y su facilidad de uso (puesto que están inmersos en la sociedad de la tecnología) favorece que el estudiante se mantenga activo y motivado durante el proceso de aprendizaje, ya que es el mismo individuo el que elige qué y cómo aprender (Anderson, 2016). Además, el profesor va cediendo terreno a favor del alumno que va logrando más autonomía e independencia en su aprendizaje, siendo en última instancia consciente de lo adquirido en el proceso (Fernández, 2006; Munuera y Navarro, 2015). Por tanto, la finalidad es ayudar al alumno a desarrollarse, propiciando el crecimiento de habilidades, destrezas y conocimientos con el objetivo final de mejorar en el desarrollo integral del alumno en consonancia con el entorno.

Actualmente, algunos docentes han desarrollado pequeñas experiencias en las que han combinado TIC's y el trabajo y evaluación de competencias transversales (Echazarreta et al., 2009; Córdova et al., 2015; Munuera y Navarro, 2015). Por ejemplo, Lucena (2016) establece que mediante su combinación, se ha logrado una participación mayor, más eficaz, fluida y efectiva por parte del alumnado. Para todos, la evaluación de competencias constituye una actividad relevante en el proceso educativo, siendo este proceso facilitado por el uso de TIC's ya que en este caso, cabe la posibilidad de que el alumno pueda constatar que efectivamente adquiere las competencias transversales comparándose con un estado inicial que es capaz de medirse mediante el uso de TIC's.

Con todo ello, y dentro del entorno UPV, este estudio propone la combinación del trabajo en competencias transversales y su evaluación mediante una TIC avanzada y que está disponible en la plataforma PoliformaT: Lesson builder.

Lesson builder es una herramienta desarrollada recientemente por Sakai e incorporada muy recientemente por nuestra universidad a la plataforma PoliformaT. Esta permite estructurar los contenidos con el objetivo de guiar a los alumnos a través de actividades y tareas de aprendizaje (Duke University, 2013). Aunque a priori, parezca que Lessons es una herramienta únicamente para planificar, esta tiene una virtud que la hace especialmente buena para la evaluación de competencias: Lessons permite la inclusión de recursos didácticos (videos, apuntes, etc.), pero además permite la interacción entre alumno-profesor y alumno-alumno. Por tanto, Lessons tiene un aliciente más a las tradicionales TIC's, y es que además de permitir la secuenciación de la docencia, permite interactuar en un mismo espacio entre todos los actores del proceso (favoreciendo el enriquecimiento en conocimientos y aprendizaje dispuesto de modo accesible en la herramienta), con la ventaja de que es utilizable de forma inmediata por todos los profesores de la UPV, sin ninguna inversión adicional.

\section{Objetivos}

El objetivo de este estudio es diseñar un modelo estándar, guía metodológica o plantilla de evaluación de competencias con el fin de trabajar y testar las competencias transversales, mediante la herramienta avanzada Lessons (Sakai), que está disponible en PoliformaT.

(c)) BY-NC-ND 2017, Universitat Politècnica de València 
Los objetivos secundarios de este estudio son:

- Definir, generar y evaluar un modelo estándar o una guía metodológica para trabajar y evaluar competencias mediante la herramienta Lessons con la finalidad de extrapolarlo a cualquier asignatura impartida en la Universidad.

- Testar la utilidad de dicho modelo en diversas escuelas y tamaño de grupos de aula para las competencias transversales

- Fomentar la participación activa y la motivación del estudiante al intentar aumentar la percepción propia en el proceso de adquisición de competencias.

\section{Desarrollo de la Innovación}

El presente trabajo se ha desarrollado en 10 asignaturas que comprenden unos 730 alumnos matriculados. Estas asignaturas pertenecen a diferentes escuelas o facultades y se imparten en diferentes titulaciones oficiales de la UPV (tabla 1).

Tabla 1: Asignaturas y titulaciones incluidas en el estudio.

\begin{tabular}{|c|c|c|c|c|c|c|c|}
\hline \multirow{2}{*}{ Titulación } & \multirow{2}{*}{$\begin{array}{c}\mathrm{N}^{\circ} \\
\text { asignaturas }\end{array}$} & \multicolumn{4}{|c|}{ Curso } & \multirow{2}{*}{ Master } & \multirow{2}{*}{ Doctorado } \\
\hline & & 1 & 2 & 3 & 4 & & \\
\hline $\begin{array}{l}\text { Grado de Ing. Forestal y del Medio } \\
\text { Natural }\end{array}$ & 1 & $\mathrm{X}$ & & & & & \\
\hline $\begin{array}{l}\text { Grado de Ing. Agroalimentaria y del } \\
\text { Medio Rural }\end{array}$ & 3 & $\mathrm{X}$ & & $\mathrm{X}$ & $\mathrm{X}$ & & \\
\hline $\begin{array}{l}\text { Grado en Ciencia y Tecnología de los } \\
\text { Alimentos }\end{array}$ & 1 & & & $\mathrm{X}$ & & & \\
\hline $\begin{array}{l}\text { Grado de Ing. de Sistemas de } \\
\text { Telecomunicación, Sonido e Imagen }\end{array}$ & 2 & & & $\mathrm{XX}$ & & & \\
\hline $\begin{array}{l}\text { Master Universitario en Ingeniería de } \\
\text { Telecomunicación }\end{array}$ & 2 & & & & & $\mathrm{X}$ & \\
\hline Master Universitario en Edificación & 1 & & & & & $\mathrm{X}$ & \\
\hline Escuela de Doctorado & 1 & & & & & & $\mathrm{X}$ \\
\hline
\end{tabular}

Los 8 profesores que componen GRIPAU, establecieron para cada una de las asignaturas las competencias transversales a trabajar mediante Lessons. De este modo las asignaturas y su breve descripción se adjuntan en la tabla 2.

Tabla 2: Asignaturas y competencias trabajadas

\begin{tabular}{|c|c|c|}
\hline Asignatura & Descripción & $\begin{array}{c}\text { Competencia } \\
\text { transversal }\end{array}$ \\
\hline $\begin{array}{c}\text { Geología, Edafología y } \\
\text { Climatología } \\
\text { (En dos grados) }\end{array}$ & $\begin{array}{l}\text { Asignatura de ler curso de los grados de } \\
\text { Ing. Agronómica y Forestal en la que los } \\
\text { alumnos deben de comprender la } \\
\text { génesis, composición y propiedades de } \\
\text { los suelos formados en diferentes }\end{array}$ & СТ.09 \\
\hline
\end{tabular}




\begin{tabular}{|c|c|c|}
\hline & condiciones ambientales. & \\
\hline $\begin{array}{c}\text { Recuperación y } \\
\text { Restauración de Suelos } \\
\text { Degradados }\end{array}$ & $\begin{array}{l}\text { Asignatura de } 40 \text { curso en la que los } \\
\text { alumnos deben identificar los factores } \\
\text { que determinan la degradación del suelo, } \\
\text { poniendo especial atención en los } \\
\text { procesos de erosión hídrica. }\end{array}$ & $\begin{array}{l}\text { СТ.09 y } \\
\text { СТ.07 }\end{array}$ \\
\hline $\begin{array}{l}\text { Sistemas y Servicios de } \\
\text { Transmisión por Radio }\end{array}$ & $\begin{array}{l}\text { Asignatura del 1er curso del Máster de } \\
\text { Ingeniería de Telecomunicación. Está } \\
\text { orientada hacia el diseño de sistemas de } \\
\text { radiocomunicaciones. }\end{array}$ & $\begin{array}{l}\text { СТ.06 y } \\
\text { СТ.08 }\end{array}$ \\
\hline Carrera Investigadora & $\begin{array}{l}\text { Curso Transversal de Doctorado, que se } \\
\text { imparte utilizando metodología on-line. } \\
\text { Los alumnos planifican su futura carrera } \\
\text { profesional en el ámbito de la } \\
\text { investigación. }\end{array}$ & CT.11 \\
\hline $\begin{array}{l}\text { Acondicionamiento } \\
\text { Acústico }\end{array}$ & $\begin{array}{l}\text { Asignatura de Master, en la que los } \\
\text { alumnos desarrollan un proyecto acústico } \\
\text { de un centro docente. }\end{array}$ & CT.05 \\
\hline $\begin{array}{c}\text { Tecnología de la Producción } \\
\text { Animal }\end{array}$ & $\begin{array}{l}\text { Asignatura de } 3 \text { er curso que pretende } \\
\text { abordar todos los aspectos necesarios } \\
\text { para diseñar alojamientos ganaderos. }\end{array}$ & $\begin{array}{l}\text { CT.05 y } \\
\text { CT.13 }\end{array}$ \\
\hline $\begin{array}{l}\text { Equipos y Subsistemas de } \\
\text { Comunicaciones }\end{array}$ & $\begin{array}{l}\text { Asignatura de master que diseñar } \\
\text { diferentes tipos de antenas y determinar } \\
\text { sus contextos de aplicación, así como } \\
\text { integrar componentes de comunicaciones } \\
\text { en la banda de microondas. }\end{array}$ & СТ.03 \\
\hline Sistemas de TV y Video & $\begin{array}{l}\text { Asignatura de grado que pretende } \\
\text { profundizar en la televisión analógica y } \\
\text { digital }\end{array}$ & $\begin{array}{l}\text { СТ.03 y } \\
\text { СТ.12 }\end{array}$ \\
\hline Comunicaciones ópticas & $\begin{array}{l}\text { Asignatura de } 3 \text { er curso que define los } \\
\text { fundamentos de los sistemas de } \\
\text { comunicaciones ópticas }\end{array}$ & $\begin{array}{l}\text { СТ.03 y } \\
\text { CT.12 }\end{array}$ \\
\hline Salud Pública & $\begin{array}{l}\text { Asignatura de } 3 \text { er curso que introduce el } \\
\text { ámbito de la protección y promoción de } \\
\text { la Salud Pública. }\end{array}$ & CT.01 \\
\hline \multicolumn{3}{|c|}{$\begin{array}{l}\text { CT.01. Comprensión e integración; CT.02. Aplicación y pensamiento práctico; CT.03. Análisis y resolución de } \\
\text { problemas; CT.04. Innovación, creatividad y emprendimiento ; CT.05. Diseño y Proyecto; CT.06. Trabajo en } \\
\text { equipo y liderazgo; CT.07. Responsabilidad ética, profesional y medio ambiental; CT.08. Comunicación efectiva; } \\
\text { CT.09. Pensamiento crítico; CT.10 Planificación y gestión del tiempo; CT.11. Aprendizaje permanente; CT.12. } \\
\text { Conocimiento de problemas contemporáneos y CT.13. Instrumentación específica. }\end{array}$} \\
\hline
\end{tabular}

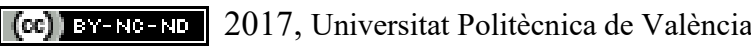


Una vez seleccionadas las competencias a trabajar se estableció el guion a desarrollar en la plataforma Lessons para cada actividad. La plantilla base tenía 3 apartados: Introducción, desarrollo de la actividad y Evaluación. En la Introducción se preguntaría a los alumnos sobre si conocían el proyecto institucional de la UPV, así como si conocían la competencia que iban a trabajar (Figura 1). En el caso de contestar negativamente a dicha cuestión eran redirigidos a la página web institucional de competencias transversales de la UPV, o tenían que ver el vídeo de competencias que el ICE había preparado para su mejor comprensión.

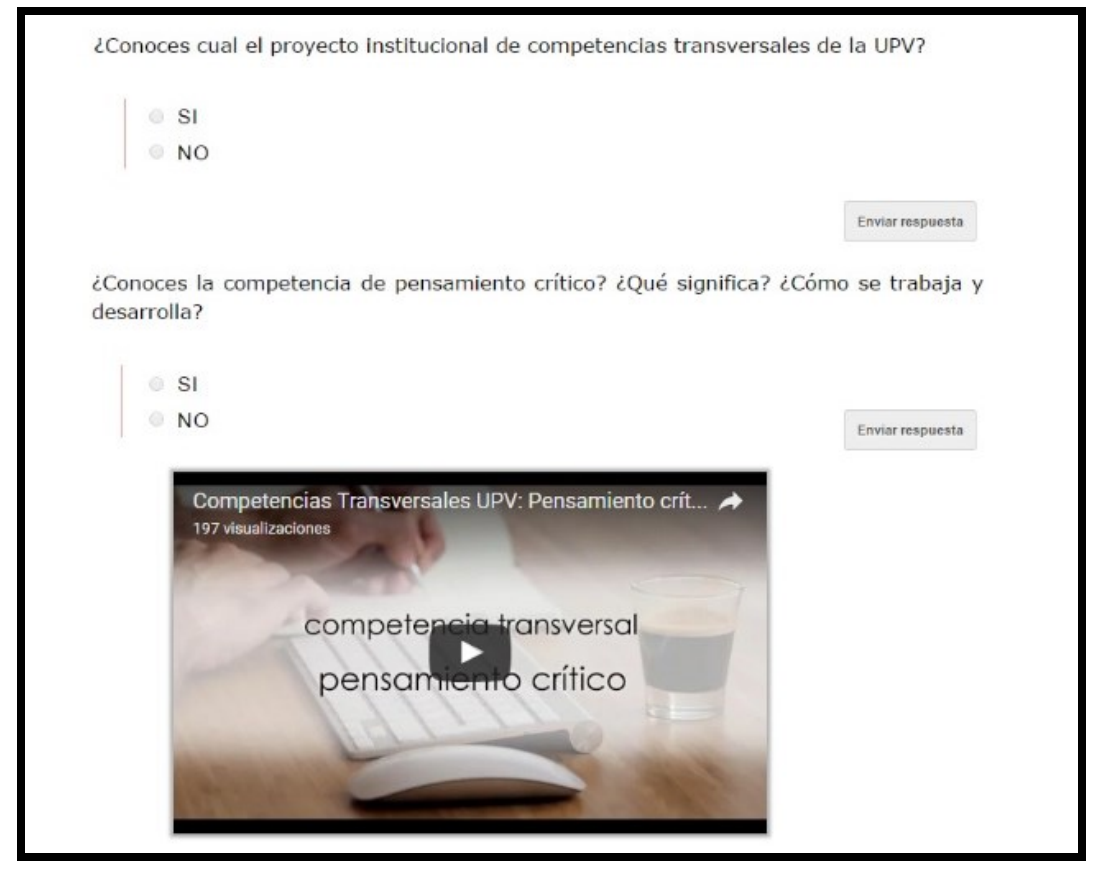

Figura 1: Preguntas iniciales en la plantilla de evaluación de competencias transversales (Lessons)

Una vez completados estos apartados, se contextualizaba al alumno con un link a la guía docente, exponiéndole que se le iba a exigir y el nivel de dominio en el que íbamos a trabajar la competencia. Posteriormente, se introdujo una encuesta sobre el estado inicial de la adquisición de la competencia en base a los ítems del nivel de dominio trabajado. Con estas cuestiones se pretendía que el alumno se autoevaluara, para posteriormente con el resultado adquirido al realizar la actividad, pudiera producirse un feedback entre profesor y alumno.

Una vez completadas las preguntas de nivel de domino, se procedía a cambiar de sección. En este caso se completó la sección Desarrollo de la actividad, en la que se describía la actividad a realizar por parte del alumno. Antes de su realización, se subió la rúbrica a emplear por el profesorado en la corrección de la actividad para que el alumno supiera cuales eran los criterios de corrección. Tras ver la rúbrica, el alumno tenía que realizar la actividad que cada profesor estableció de acuerdo a los contenidos propios de la asignatura. Las actividades propuestas se establecen en la tabla 3. 
Tabla 3: Actividades desarrolladas

\begin{tabular}{|c|c|}
\hline Asignatura & Actividad \\
\hline $\begin{array}{l}\text { Geología, Edafología y } \\
\text { Climatología }\end{array}$ & $\begin{array}{l}\text { Situación real planteada en clase donde el alumno tenía } \\
\text { que decidir cual de las dos opciones planteadas por dos } \\
\text { alumnos diferentes era la correcta. }\end{array}$ \\
\hline $\begin{array}{c}\text { Recuperación y } \\
\text { Restauración de Suelos } \\
\text { Degradados }\end{array}$ & $\begin{array}{l}\text { Caso práctico en el que los alumnos tienen que aplicar la } \\
\text { ética profesional y el pensamiento crítico al valorar los } \\
\text { datos reales de un caso de degradación/erosión. }\end{array}$ \\
\hline $\begin{array}{l}\text { Sistemas y Servicios de } \\
\text { Transmisión por Radio }\end{array}$ & $\begin{array}{l}\text { Trabajo en grupo realizado durante } 11 \text { sesiones, para } \\
\text { diseñar un sistema real. Se concluye con una redacción de } \\
\text { memoria y presentación oral de los resultados finales }\end{array}$ \\
\hline Carrera Investigadora & $\begin{array}{l}\text { Búsqueda de información sobre Carrera Investigadora, } \\
\text { difusión en los foros de la asignatura y debate con los } \\
\text { compañeros. Realización de un trabajo sobre acreditación } \\
\text { como Ayudante Doctor. }\end{array}$ \\
\hline $\begin{array}{l}\text { Acondicionamiento } \\
\text { Acústico }\end{array}$ & $\begin{array}{l}\text { Desarrollo de un proyecto acústico sobre un centro } \\
\text { docente, aplicando la metodología de proyecto de cara a la } \\
\text { resolución de una situación compleja con múltiples } \\
\text { variables y criterios diferentes. }\end{array}$ \\
\hline $\begin{array}{c}\text { Tecnología de la Producción } \\
\text { Animal }\end{array}$ & $\begin{array}{l}\text { Desarrollo de un proyecto de granja avícola y un proyecto } \\
\text { de granja porcina. Los alumnos deberán seleccionar una } \\
\text { ubicación adecuada y diseñar la granja de acuerdo con los } \\
\text { condicionantes de esa localización, respetando los } \\
\text { condicionantes legales, considerando las necesidades de } \\
\text { cada especie y teniendo en cuenta las recomendaciones } \\
\text { generales para facilitar el manejo de la misma. }\end{array}$ \\
\hline $\begin{array}{l}\text { Equipos y Subsistemas de } \\
\text { Comunicaciones }\end{array}$ & $\begin{array}{l}\text { Diseño de un proyecto en el cual el alumno debe analizar y } \\
\text { resolver problemas referentes a la asignatura }\end{array}$ \\
\hline Sistemas de TV y Video & Trabajos y resolución de problemas \\
\hline Comunicaciones ópticas & $\begin{array}{l}\text { Trabajos y resolución de problemas sobre la viabilidad de } \\
\text { un sistema de comunicación óptima }\end{array}$ \\
\hline Salud pública & $\begin{array}{l}\text { Resolución de una situación real planteada que presenta } \\
\text { problemas de solución múltiple. }\end{array}$ \\
\hline
\end{tabular}

Por último, se establece la sección de Evaluación que consistía en la evaluación del profesorado (en algunos casos), o por pares (en otros) de la actividad realizada por los alumnos. La calificación final se obtenía en base a una serie de letras que indicaban el grado de consecución de la competencia trabajada: A (Excelente/ejemplar); B (Bien /adecuado); C (En desarrollo) y D (No alcanzado). 
De este modo, se establecía un feedback con el alumno que comparaba los resultados de su test de autoevaluación final con el real (alcanzado con el desarrollo de la actividad). Por otro lado, el alumnado también tuvo que contestar a 3 simples preguntas para evaluar Lessons y su idoneidad.

\section{Resultados}

Desde el punto de visto de los profesores, al iniciar el Proyecto, conocían la herramienta Lessons un $33 \%$, por haberla utilizado previamente o haber seguido un curso de formación previo. Esto ha implicado a un aprendizaje interno, en el que el profesorado conocedor de la herramienta ha vehiculado la información hacia los profesores que desconocían dicha herramienta. En este caso, para el resto de los participantes en el Grupo se realizaron dos sesiones de formación, con el apoyo de los profesores que ya conocían la herramienta. Resultó especialmente útil el disponer de una plantilla inicial, la cual ha sido mejorada por el profesorado que ya conocía la herramienta, y por aquel profesorado que ha realizado el curso de Lessons del Instituto de Ciencias de la Educación.

En general la implementación se ha realizado en algunas asignaturas para la totalidad de los contenidos, mientras que en otras solo se ha desarrollado Lessons para trabajar la competencia transversal seleccionada. La valoración por parte de los miembros del grupo de innovación de Lessons es muy favorable, estando prevista la migración de los Contenidos de las herramientas anteriores en un período relativamente breve. En este sentido, aquellos alumnos que tenían todos los contenidos y tareas de la asignatura ya estructurados en Lessons, la aceptación de la herramienta ha sido mucho mejor que aquellos que solo han desarrollado la competencia transversal como veremos a continuación.

También es de destacar que el estudio se ha aplicado de forma satisfactoria en grupos de diferente tamaño y diferentes escuelas y la respuesta ha sido homogénea a todas estas variables. Lessons por tanto facilita el manejo de grupos de pequeño, mediano y gran tamaño, siendo de aplicación multidisciplinar la plantilla creada para el desarrollo de la actividad.

Por lo que respecta a los alumnos, del total que realizaron las actividades de las asignaturas, el $80 \%$ como media considera que tiene un nivel de desarrollo de la competencia transversal bueno o excelente. No obstante, tras realizar la actividad, únicamente un $46 \%$ de los alumnos presentan dicho estatus, siendo únicamente un $23 \%$ los que realmente tienen un nivel de adquisición de las competencias excelente. En este sentido, el alumno comprobó que el nivel competencial que tenía no se correspondía con el real, viéndose en el feedback con los alumnos de forma individual el porqué de dicho desfase. 
Por lo que respecta al conocimiento de proyecto institucional de la UPV, un $18.6 \%$ dicen no conocer el proyecto de competencias transversales UPV, siendo el $84 \%$ de media aquellos que conocen la competencia que van a trabajar puesto que la están trabajando en otras asignaturas o porque han oído hablar de ellas en otras asignaturas o en las jornadas de acogida (en los alumnos de primer curso). En este sentido, han valorado positivamente la redirección con links de aquellos conceptos o recursos que desconocían. No obstante, un 12 $\%$ asegura que dichos links están bien para tenerlos como recurso pero que le tomaron poca importancia por las tareas restantes de otras asignaturas y el tiempo para la realización.

Por último, destacar que el $81.6 \%$ de los alumnos creen que es necesario la autoevaluación previa a la realización de las actividades pues fomenta el conocimiento propio del estado de adquisición de la competencia una vez el profesor ha evaluado dicha actividad. En este sentido el $56 \%$ de los alumnos cree que Lessons es un buen secuenciador de contenidos. Por el contrario, en alumnos de primero el 55\% de los alumnos cree que podría haberse trabajado la competencia sin la necesidad de utilizar Lessons. No obstante, el 30\% del alumnado cree que Lessons le ha facilitado el trabajo y la adquisición de la competencia. Hay una gran diferencia entre los alumnos de primero (en el primer cuatrimestre) y los alumnos de otros cursos en cuanto al manejo de la plataforma PoliformaT, puesto que no están aún acostumbrados al entorno Lessons/PoliformaT. Además, hay que destacar la diferencia también entre los alumnos que únicamente han desarrollado la actividad de Competencia Transversal por Lessons, frente aquellos que tienen todos los contenidos de la asignatura estructurados en Lessons. En este caso, el nivel de idoneidad/satisfacción de Lessons es mayor.

\section{Conclusiones}

Como conclusión general, este estudio y el trabajo realizado por los 8 profesores que conforman este proyecto ha facilitado la creación de una plantilla efectiva para la evaluación de Competencias Transversales mediante Lessons. Así mismo, se ha testado la utilidad de la plantilla a diferentes niveles: grado, master y doctorado; grupos de tamaño pequeño, mediano y grande, y en diferentes escuelas de la UPV.

Por último, mediante Lessons y su filosofía 3.0 se ha motivado al alumnado en la realización y adquisición de competencias, puesto que de forma rápida y concreta han podido desarrollar las tareas preparadas para tal fin. Además, han participado activamente del proceso de autoevaluación y evaluación de la competencia.

\section{Referencias}

ANDERSON, M. (2016). Learning to Choose, Choosing to Learn: The Key to Student Motivation and Achievement. Association for Supervision \& Curriculum Development. 
BAIN, K. (2007). Lo que hacen los mejores profesores universitarios. Universidad de Valencia Valenca (España)

CÓRDOVA, A, et al. (2015). "Construcción de un instrumento para evaluar competencias profesionales durante la formación preclínica en Medicina". Investigación en Educación Médica 4: $145-154$

DUKE University (2013). Getting started with the Lessons tool. https://support.sakai.duke.edu/2013/05/17/lessons/ 02/03/2017

ECHAZARRETA, C, et al.. (2009). "La competencia «El trabajo colaborativo»: una oportunidad para incorporar las TIC en la didáctica universitaria. Descripción de la experiencia con la plataforma ACME (UdG) ". Revista sobre la sociedad del conocimiento $8: 1-11$.

FERNÁNDEZ, A. (2006). "Metodologías activas para la formación de competencias". Educatio siglo $X X I, 24:$ 35-56.

LUCENA, I (2015). "La aplicación de las TIC y la evaluación por competencias en el Grado de Derecho". International journal of educational research and innovation, 5: 42-54

MEZARINA, C., et al., (2014). "Aplicación de las TIC en educación superior como estrategia innovadora para el desarrollo de competencias digitales". Revista científica de tecnología educativa 1 : 88-101

MUNUERA, P, y NAVARRO, E. (2015). "Innovación en la Evaluación de Competencias Transversales. El instrumento PIAESCE”. Opción 1: 510-528

UPV (2014). Plan Estratégico UPV 2015-2020. Universitat Politècnica de València. 43p. 